Volume 2 Issue 2

December 2017

\title{
Treat the patient, not the machine...always true? A case report of silent myocardial infarction.
}

Damien Gaumont HDipEMT

National Ambulance Service, Ireland.

Paramedic Studies, Graduate Entry Medical School, University of Limerick, Ireland.

\section{Recommended Citation}

Gaumont, D. Treat the patient, not the machine...always true? A case report of silent myocardial infarction. Irish Journal of Paramedicine. 2017 Dec; 2(2).

This is an Open Access article distributed under the terms of the Creative Commons Attribution-Non-Commercial-ShareAlike 4.0 International (http://creativecommons.org/licenses/by-nc-sa/4.0/), which permits use, distribution, and reproduction in any medium, provided the original work and any attributes thereof are properly cited, are distributed under the same licence, and that the work is not used for commercial purposes.

Follow the Irish Journal of Paramedicine online at www.irishparamedicine.com, on Twitter (@irishjparamed) and on Facebook. 
CASE REPORT

\title{
Treat the patient, not the machine...always true? A case report of silent myocardial infarction.
}

\author{
Damien Gaumont HDipEMT AP
}

\section{National Ambulance Service, Limerick, Ireland. 2. Paramedic Studies, Graduate Entry Medical School, University of Limerick, Ireland. \\ Received: 09 Nov $2017 \quad$ Accepted: 03 Dec $2017 \quad$ Published: 15 Dec 2017 \\ Correspondence: Damien Gaumont, National Ambulance Service, Limerick, Ireland. Email: damien.gaumont@gmail.com}

\section{Case Summary}

After an unusual prehospital ST-segment elevated myocardial infarction (STEMI) presentation, with a completely asymptomatic patient, a literature review was performed investigating the incidence and pathophysiology of true asymptomatic myocardial infarction.

\section{Background}

From their early days in education, paramedics are reminded not to be machine orientated, but to look at, and treat, the patient in front of them. Yet, the following case challenges this mantra. This is a report of a prehospital presentation of a 12-Lead ECG showing classic characteristics of a STEMI, obtained from a completely asymptomatic patient.

\section{Case Presentation}

At 16:00 on a week day, a Paramedic/Advanced Paramedic crew was dispatched to a general practitioner's (GP) surgery for a reported STEMI. On arrival the doctor took the crew aside and explained the patient's peculiar presentation. A 54 year old male woke up with severe pain in his left wrist at 03:00 with associated diaphoresis. The symptoms subsided rapidly and the patient returned to sleep. The patient presented himself to the surgery the following day, completely asymptomatic, after completing his daily activities and walk. The only abnormality found was a slight hypertension (155/110 mmHg). Initial 12-Lead ECG showed sinus rhythm at $80 \mathrm{bpm}$, with ST elevation in II, III and aVF, and ST depression in I, aVL, V2 and V3 (Figure 1). The GP administered $300 \mathrm{mg}$ of aspirin as a precaution.

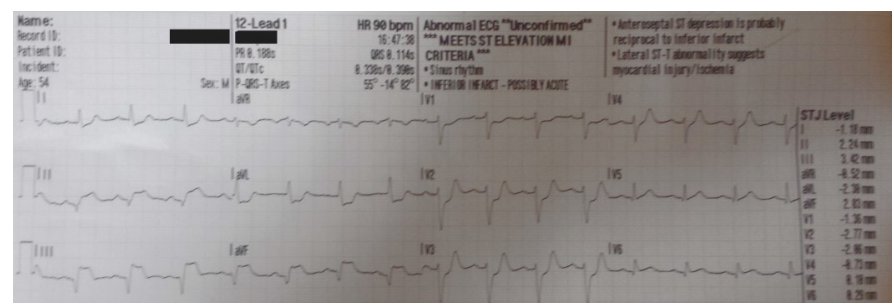

Figure 1. 12-Lead ECG

The patient had a previous history of hyperlipidaemia which was under control with diet only, smoked 15 cigarettes a day for 30 years and drank alcohol occasionally. His family history did not reveal any heart disease. He was working shift the previous three days. Vital signs were obtained by the crew and the 12 lead ECG repeated.

\section{Investigations}

The patient appeared well groomed and appropriately dressed, with no visible excess weight. He had a heart rate of $85 \mathrm{bpm}$ with a blood pressure of $155 / 108 \mathrm{mmHg}$, a respiratory rate of $14 \mathrm{bpm}$, unlaboured with an $\mathrm{SpO} 2$ of $97 \%$ on room air.
Physical examination was unremarkable. The patient was alert, fully orientated without any anxiety, and wondering what "all the fuss was about".

The repeat 12-Lead ECG confirmed the GP's findings and a right-sided ECG showed slight ST elevation and inverted T waves on V4R, V5R and V6R (Figure 2).

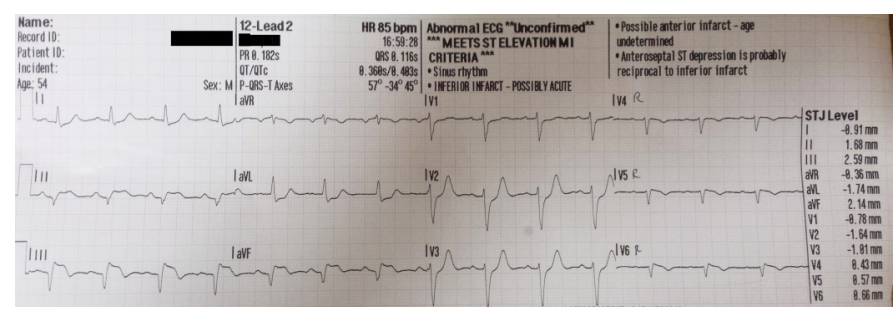

Figure 2. Right-sided 12-Lead ECG

\section{Differential diagnosis}

All clinicians at scene were at a loss to find another explanation for the ECG findings, bar unusual pathophysiology, or conduction pathway anomaly. It was decided to heed the obvious diagnosis and treat as an Acute Coronary Syndrome presentation.

\section{Management}

Aspirin had been already administered, and oxygen, nitrates or morphine were not indicated. The Cardiology Department was contacted for direct access to cardiac catheterisation lab (bypassing the Emergency Department-ED), and clinical advice.

Direct access to the cath lab was declined due to the lack of pain in a non-diabetic, and the team being already engaged in a procedure. A cardiology registrar reviewed the ECG in the Emergency Department and directed the patient towards the cardiac catheterisation lab. Angiogram showed small occlusion of the right coronary artery midway, which was subsequently stented.

\section{Outcome and follow-up}

The patient was discharged several days later with GP follow up, and successfully returned to work.

\section{Discussion}

Education and experience has imparted to us than not all myocardial infarctions present with "classic" crushing chest pain radiating down the left arm. In fact, between $10-20 \%$ of patients with Acute Myocardial Infarction - in particular women, diabetics, older and heart transplant recipients - do not experience any chest pain. Sudden shortness of breath, sudden loss of consciousness, sudden drop of blood pressure, confusion, stroke, indigestion, malaise, fatigue, or just not feeling well, are some of the concerning symptoms. Women may experience nausea, light-headedness, epigastric burn, sudden weakness, or tiredness (1).

The incidence of patients being completely 
asymptomatic is rare and poorly understood (2). They are often found in studies following groups of patients whom reviewed ECG reveal pathological Q waves. In their literature review of Silent Myocardial Infarction (SMI) discovered during routine investigations, Cohn et al. (2) discovered an incidence of $2.5 \%$ in the less of 60 year old had no symptoms; $50 \%$ of them had a coronary lesion. The figure of SMI rose to $10 \%$ for the over 70 year old. Valensi et al.'s (3) literature review of the incidence of SMI, suggests the prevalence of SMI in non-diabetic patients with the absence of cardiovascular diseases (as is our patient in this case study) to be between $0.3 \%$ and $5.4 \%$ depending on the population's age. This confirms the findings of an older, small study of 259 routine ECG (of white males only) showing Q waves, $3.7 \%$ were completely asymptomatic (4).

Davis et al. (5) reviewed the UK Prospective Diabetes Study (UKPDS 1991) and found than one in six patient newly diagnosed with type 2 diabetes had evidence of SMI. DeLucas et al.'s (6) comparison study of diabetic versus nondiabetic patients, suggested that prevalence of SMI was twice as likely in the diabetic patient.

Associated risk factors in the diabetic patient increase the risks of SMI to $10 \%$ with peripheral neuropathy and a staggering $30 \%$ with coronary or peripheral artery diseases (3).

\section{Learning points / take home messages}

This patient presentation puzzled not only the patient, but also all medical personnel present at scene. Because of the obvious ECG findings, expert help was sought immediately in the form of the STEMI line, and the cardiology team met the patient on arrival at the Emergency Department.

This case challenges the mantra "Treat the patient, not the machine".

\section{References}

1. Caroline, N. L. Emergency Care on the Street (sixth ed.). (B. Elling, \& A. N. Pollack, Eds.) 2008. Sudbury, MA: Jones and Bartlett Publishers.

2. Cohn, P. F., Fox, M. K., \& Daly, C. Silent Myocardial Ischemia. Circulation, 2003;10(108),1263-1277.

3. Valensi, P., Lorgis, L., \& Cottin, Y. Prevalence, incidence, predictive factors and prognosis of silent myocardial infarction: a review of the literature. Archives of Cardiovascular Disease, 2011;178-188.

4. Margolis, J. R., Kannel, W. B., Feinleib, M., Dawber, T. R., \& McNamara, P. M. Clinical features of unrecognized myocardial infarction: Eighteen year follow-up: The Framingham study. The American Journal of Cardiology, 1973;32(1), 1-7.

5. Davis, T. M., Coleman, R., \& Holman, R. R. Prognostic Significance of silent myocardial infarction in newlydiagnosed type 2 diabetes: UKPDS79. Circulation, 2013; [on line].

6. DeLucas, A. J., Kaplan, S., Aronow, W. S., Akoybyan, A., $\&$ Weiss, M. B. Comparison of prevalence of unrecognised myocardial infarction and of silent myocardial infarction detected by treadmill exercise Sestamibi stress test in patient with versus without diabetes mellitus. The Journal of American Cardiology, 2006;98(8), 1045-1046.

Editor's note: Higher quality ECGs could not be sourced. Author contributions: DG was responsible for the writing of the manuscript. Copyediting was performed by IJP staff.
Conflict of interest: No conflicts of inter est were declared. Provenance and review: Not commissioned, peerreviewed.

This is an Open Access article distributed under the terms of the Creative Commons Attribution-Non-Commercial-ShareAlike 4.0 International (http:// creativecommons.org/licenses/by-nc-sa/4.0/), which permits use, distribution, and reproduction in any medium, provided the original work and any attributes thereof are properly cited, are distributed under the same licence, and that the work is not used for commercial purposes. Content copyright remains with the authors, who grant the IJP a licence to reuse and distribute. 